\title{
ESTIMATES AND RIGIDITY FOR STABLE SOLUTIONS TO SOME NONLINEAR ELLIPTIC PROBLEMS
}

\author{
PIETRO MIRAGLIO
}

(Received 28 May 2020; first published online 10 September 2020)

2020 Mathematics subject classification: primary 35J15; secondary 35B45.

Keywords and phrases: elliptic equations, p-Laplacian, stable solutions, inequalities on hypersurfaces, nonlocal operators, Dirichlet to Neumann operator, one-dimensional symmetry, Fourier operators.

The thesis deals with elliptic partial differential equations. It is divided into two parts, the first concerning a nonlinear elliptic equation involving the $p$-Laplacian and the second focused on a nonlocal problem arising from a model for water waves.

In the first part, we study the regularity of stable solutions to the nonlinear elliptic equation involving the $p$-Laplacian

$$
-\Delta_{p} u:=\operatorname{div}\left(|\nabla u|^{p-2} \nabla u\right)=f(u) \quad \text { in } \Omega \subset \mathbb{R}^{n},
$$

where $\Omega$ is a bounded domain, $p \in(1,+\infty)$ and $f$ is a $C^{1}$ nonlinearity. This equation is the nonlinear version of the widely studied semilinear elliptic equation $-\Delta u=f(u)$ in a bounded domain $\Omega \subset \mathbb{R}^{n}$. Stable solutions to semilinear equations have very recently been proved to be bounded, and therefore smooth, in dimension $n \leq 9$ (see [2]). This result is optimal, since examples of unbounded stable solutions are well known in dimension $n \geq 10$. Moreover, the results in [2] give a complete answer to a long-standing open problem about the regularity of extremal solutions to $-\Delta u=\lambda f(u)$.

We investigate the boundedness of stable solutions to (1) up to dimension $n$ with $n<p+4 p /(p-1)$. If $n \geq p+4 p /(p-1)$, examples of unbounded stable solutions are known even in the unit ball. In the radial case or under strong assumptions on the nonlinearity, stable solutions to (1) are proved to be bounded for $n<p+4 p /(p-1)$.

In the thesis (see [7]), we prove a new $L^{\infty}$ a priori estimate for stable solutions to (1), under a new condition on $n$ and $p$, which is optimal in the radial case and more restrictive in the general one. However, it improves the known results in the

The thesis was written under the supervision of Enrico Valdinoci of the University of Western Australia and a significant part of the work was conducted in Australia. The degree was approved jointly by Università degli Studi di Milano (Milan, Italy) and the Universitat Politècnica de Catalunya (Barcelona, Spain) on 28 January 2020.

(C) 2020 Australian Mathematical Publishing Association Inc. 
field and it is the first example of a technique providing both a result in the nonradial case and the optimal result in the radial case. To establish this result, we extend a technique developed by Cabré [1] for the classical case of the problem, with $p=2$, to the framework of the $p$-Laplacian. The strategy is based on a Hardy inequality on the level sets of the solution and on a geometric inequality for stable solutions to (1).

We also investigate Hardy-Sobolev inequalities on hypersurfaces of Euclidean space, all of them involving a mean-curvature term and having universal constants independent of the hypersurface (see [3]). Our motivation comes from several applications of these inequalities to the study of a priori estimates for stable solutions, in both the semilinear and the nonlinear cases.

Our first Hardy inequality originates from an application to the regularity of stable solutions for semilinear elliptic equations. We use this result in the proof of our $L^{\infty} a$ priori estimate for stable solutions to (1). Our second Hardy inequality is proved using the 'ground-state' substitution, a technique based on exploiting a specific positive solution of the Euler-Lagrange equation of the energy functional associated with the inequality. We then use a refinement of this procedure, combined with a Poincaré inequality with weights, to obtain a Hardy-Poincaré inequality on hypersurfaces. This seems to be the first use of the ground-state substitution on hypersurfaces of $\mathbb{R}^{n+1}$.

In the second part of this thesis, we deal with a Dirichlet to Neumann problem arising in a water waves model. Considering a slab of fixed height $\mathbb{R}^{n} \times[0,1]$, a smooth bounded function $u$ defined on $\mathbb{R}^{n}$ and a parameter $a \in(-1,1)$, we are interested in studying the system of equations

$$
\begin{cases}\operatorname{div}\left(y^{a} \nabla v\right)=0 & \text { for } x \in \mathbb{R}^{n}, y \in(0,1), \\ v_{y}(x, 1)=0 & \text { for } x \in \mathbb{R}^{n}, y=1, \\ v(x, 0)=u(x) & \text { for } x \in \mathbb{R}^{n}, y=0, \\ -\lim _{y \rightarrow 0} y^{a} v_{y}=f(v) & \text { for } x \in \mathbb{R}^{n}, y=0\end{cases}
$$

This system can also be reformulated as a nonlocal problem on the component endowed with the Dirichlet datum (see [6]). Indeed, we can define the Dirichlet to Neumann operator $\mathcal{L}_{a}$ as

$$
\mathcal{L}_{a} u(x):=-\lim _{y \rightarrow 0} y^{a} v_{y}(x, y)
$$

and study the nonlocal equation $\mathcal{L}_{a} u=f(u)$ in $\mathbb{R}^{n}$.

Problem (2) was first studied in the case $a=0$ by de la Llave and Valdinoci [5]. Their main result is a Liouville theorem that yields the one-dimensional symmetry of monotone solutions to problem (2) with $a=0$ and $n=2$. In the thesis (see also [4]), we extend the Liouville theorem in [5] from $a=0$ to all the fractional parameters $a \in(-1,1)$, also considering the wider class of stable solutions to (2) instead of monotone solutions. As a consequence, we obtain the one-dimensional symmetry of stable solutions to (2) in dimension $n=2$ for every parameter $a \in(-1,1)$.

Moreover, we consider the three-dimensional case of problem (2) with $a \in(-1,1)$, establishing sharp energy estimates for both the energy minimisers and the monotone 
solutions to (2). These estimates lead to the one-dimensional symmetry of these classes of solutions when $n=3$ for every $a \in(-1,1)$, by an application of the Liouville theorem mentioned above.

For this nonlocal problem, we also investigate the nature of the operator $\mathcal{L}_{a}$ defined in (3) (see [8]). First, we deduce its expression as a Fourier operator for every $a \in(-1,1)$, which was previously known only for $a=0$. As a result, we show that the Fourier symbol of $\mathcal{L}_{a}$ is asymptotic to that of the fractional Laplacian for high frequencies, but similar to the symbol of the classical Laplacian for small frequencies. This behaviour highlights the mixed nature of the operator $\mathcal{L}_{a}$, which is nonlocal, but not of purely fractional type, and structurally depends on the fractional parameter $a$. We further investigate this aspect by studying the $\Gamma$-convergence of the energy functional associated to the equation $\mathcal{L}_{a} u=W^{\prime}(u)$, where $W$ is a double-well potential.

Specifically, we prove the $\Gamma$-convergence of the energy functional to a limit that corresponds to a mere interaction energy when $a \in(0,1)$ and to the classical perimeter when $a \in(-1,0]$. In terms of the corresponding fractional parameter $s=(1-a) / 2$, this dichotomy reflects a purely nonlocal behaviour when $s \in(0,1 / 2)$ and purely classical asymptotics when $s \in[1 / 2,1)$. The threshold $s=1 / 2$, as well as the $\Gamma$-limit behaviour for the regime $s \in[1 / 2,1)$, is common to other nonlocal problems, but the limit functional in the strongly nonlocal regime $s \in(0,1 / 2)$ appears to be new and structurally different from already well-known nonlocal energy functionals.

\section{References}

[1] X. Cabré, 'A new proof of the boundedness results for stable solutions to semilinear elliptic equations', Discrete Contin. Dyn. Syst. 39 (2019), 7249-7264.

[2] X. Cabré, A. Figalli, X. Ros-Oton and J. Serra, 'Stable solutions to semilinear elliptic equations are smooth up to dimension 9', Acta Math. 224 (2020), 187-252.

[3] X. Cabré and P. Miraglio, 'Universal Hardy-Sobolev inequalities on hypersurfaces of Euclidean space', Preprint, arXiv:1912.09282 (2019).

[4] E. Cinti, P. Miraglio and E. Valdinoci, 'One-dimensional symmetry for the solutions of a three-dimensional water wave problem', J. Geom. Anal. 30 (2020), 1804-1835.

[5] R. de la Llave and E. Valdinoci, 'Symmetry for a Dirichlet-Neumann problem arising in water waves', Math. Res. Lett. 16 (2009), 909-918.

[6] S. Dipierro, P. Miraglio and E. Valdinoci, 'Symmetry results for the solutions of a partial differential equation arising in water waves', in: 2018 MATRIX Annals, MATRIX Book Series, 3 (Springer, Cham, 2020).

[7] P. Miraglio, 'Boundedness of stable solutions to nonlinear equations involving the $p$-Laplacian', $J$. Math. Anal. Appl. 489 (2020), to appear.

[8] P. Miraglio and E. Valdinoci 'Energy asymptotics of a Dirichlet to Neumann problem related to water waves', Preprint, arXiv:1909.02429 (2019).

PIETRO MIRAGLIO, Dipartimento di Matematica,

Università degli Studi di Milano, via Cesare Saldini 50, 20133, Milano, Italy

and

Universitat Politècnica de Catalunya,

Departament de Matemàtiques, Diagonal 647, 08028, Barcelona, Spain

e-mail: miraglio.pietro@gmail.com 